\title{
Modulation-mode assignment for SVD-aided and BICM-assisted spatial division multiplexing
}

\author{
Andreas Ahrens ${ }^{\mathrm{a}}$, Soon Xin $\mathrm{Ng}^{\mathrm{b}}$, Volker Kühn ${ }^{\mathrm{a}}$, Lajos Hanzo ${ }^{\mathrm{b}, *}$ \\ ${ }^{a}$ University of Rostock, Institute of Communications Engineering, Richard-Wagner-Street 31, 18119 Rostock, Germany \\ ${ }^{\mathrm{b}}$ University of Southampton, Communications Research Group, Southampton, SO17 1BJ, United Kingdom
}

\begin{abstract}
In this contribution the number of activated MIMO layers and the number of bits per symbol along with the appropriate allocation of the transmit power and the rate of the BICM error correcting codes are jointly optimized under the constraint of a given fixed data throughput. The performance investigations are carried out by computer simulations and confirmed by the EXIT charts. Our results show that not necessarily all MIMO layers have to be activated in order to achieve the best BERs.

(c) 2008 Elsevier B.V. All rights reserved.

Keywords: MIMO; Singular value decomposition; Transmit preprocessing; Bit-interleaved coded modulation; Iterative detection; Spatial division multiplexing
\end{abstract}

\section{Introduction}

Iterative demapping and decoding-aided bitinterleaved coded modulation (BICM-ID) was designed for bandwidth efficient transmission over fading channels $[1,2]$. The BICM philosophy has been extended by using different throughput modulated signal constellations and bit-to-symbol mapping arrangements, leading to the concept of bit-interleaved coded irregular modulation (BICIM) schemes, which offer an improved link adaptation capability and an increased design freedom[3]. Since the capacity of Multiple Input Multiple Output (MIMO) systems increases linearly with the number of antennas at both, the transmitter as well as the receiver side, MIMO-BICM schemes have attracted substantial attention [4,5]. However, their parameters

\footnotetext{
* Corresponding author. Tel.: +44 2380593 125; fax: +44 2380 594508.

E-mail addresses: andreas.ahrens@uni-rostock.de (A. Ahrens), sxn@ecs.soton.ac.uk (S.X. Ng), volker.kuehn@uni-rostock.de (V. Kühn), 1h@ecs.soton.ac.uk (L. Hanzo).
}

have to be carefully optimized, especially in conjunction with adaptive modulation [6-11].

Against this background, the novel contribution of this paper is that we jointly optimize the number of activated MIMO layers and the number of bits per symbol along with the appropriate allocation of the transmit power and the rate of the BICM scheme's error correcting codes used under the constraint of a given fixed data throughput and integrity. Since the "design-space" is large, a two-stage optimization technique is considered. Firstly, the uncoded Spatial Division Multiplexing (SDM) MIMO scheme is optimized, investigating the adaptive allocation of both the number of bits per modulated symbol and the number of activated MIMO layers at a fixed data rate. Secondly, the resultant uncoded systems are extended by incorporating BICM-ID, whereby both the uncoded as well as the coded systems are required to support the same user data rate within the same bandwidth. The performance investigations are carried out by computer simulations and confirmed by EXIT charts [12]. 
The remainder of this letter is organized as follows Section 2 introduces our system model and the performance metrics. In Section 3 the channel-encoded MIMO system is introduced, while our EXIT chart analysis is provided in Section 4. The associated performance results are presented and interpreted in Section 5. Finally, Section 6 provides our concluding remarks.

\section{System model and quality criteria}

When considering a non-frequency selective SDM MIMO link constituted by $n_{\mathrm{T}}$ transmit and $n_{\mathrm{R}}$ receive antennas, the system is modelled by

$\mathbf{u}=\mathbf{H} \cdot \mathbf{c}+\mathbf{w}$.

In (1), $\mathbf{u}$ is the $\left(n_{\mathrm{R}} \times 1\right)$ received vector, $\mathbf{c}$ is the $\left(n_{\mathrm{T}} \times\right.$ 1) transmitted signal vector containing the complex input symbols and $\mathbf{w}$ is the $\left(n_{\mathrm{R}} \times 1\right)$ vector of the Additive White Gaussian Noise (AWGN) having a variance of $U_{\mathrm{R}}^{2}$ for both the real and imaginary parts. Furthermore, we assume that the coefficients of the $\left(n_{\mathrm{R}} \times n_{\mathrm{T}}\right)$ channel matrix $\mathbf{H}$ are independently Rayleigh distributed with equal variance. Throughout this paper, vectors and matrices are printed in boldface roman letters. Furthermore, sequences of random variables (r.v.s) are indicated by boldface italics capital letters and their corresponding realizations by boldface italics lower case letters, respectively. The mitigation of the interference between the different antennas' data streams, which is introduced by the off-diagonal elements of the channel matrix $\mathbf{H}$, requires appropriate signal processing strategies. A popular technique is based on the singular value decomposition (SVD) of the system matrix $\mathbf{H}$, which can be written as $\mathbf{H}=$ $\mathbf{S} \cdot \mathbf{V} \cdot \mathbf{D}^{\mathrm{H}}$, where $\mathbf{S}$ and $\mathbf{D}^{\mathrm{H}}$ are unitary matrices and $\mathbf{V}$ is a real-valued diagonal matrix of the positive square roots of the eigenvalues of the matrix $\mathbf{H}^{\mathrm{H}} \mathbf{H}$ sorted in descending order. The transpose and conjugate transpose (Hermitian) of $\mathbf{D}$ are denoted by $\mathbf{D}^{\mathrm{T}}$ and $\mathbf{D}^{\mathrm{H}}$, respectively. The SDM MIMO data vector $\mathbf{c}$ is now multiplied by the matrix $\mathbf{D}$ before transmission. In turn, the receiver multiplies the received vector $\mathbf{u}$ by the matrix $\mathbf{S}^{\mathrm{H}}$. Thereby neither the transmit power nor the noise power is enhanced. The overall transmission relationship is defined as

$\mathbf{y}=\mathbf{S}^{\mathrm{H}}(\mathbf{H} \cdot \mathbf{D} \cdot \mathbf{c}+\mathbf{w})=\mathbf{V} \cdot \mathbf{c}+\tilde{\mathbf{w}}$.

Here, the channel matrix $\mathbf{H}$ is transformed into independent, non-interfering layers having unequal gains. In general, the quality of data transmission can be informally assessed by using the half vertical eye opening and the noise power at the detector's input. The resultant quality criteria per quadrature component becomes

$\varrho=\frac{(\text { Half vertical eye opening })^{2}}{\text { Noise Power }}=\frac{\left(U_{\mathrm{A}}\right)^{2}}{\left(U_{\mathrm{R}}\right)^{2}}$,

which is often used as a quality parameter [13] The relationship between the quality criteria $\varrho=$ $U_{\mathrm{A}}^{2} / U_{\mathrm{R}}^{2}$ and the bit-error probability (BER) evaluated for AWGN channels and $M$-ary Quadrature Amplitude Modulation (QAM) is given by $[11,14]$

$P_{\mathrm{e}}=\frac{2}{\log _{2}(M)}\left(1-\frac{1}{\sqrt{M}}\right) \operatorname{erfc}\left(\sqrt{\frac{\varrho}{2}}\right)$.

When applying the proposed system structure, the SVDbased equalization leads to different eye opening per MIMO layer $\ell$ and per transmitted symbol block $k$ according to

$U_{\mathrm{A}}^{(\ell, k)}=\sqrt{\xi_{\ell, k}} \cdot U_{\mathrm{s}} \ell$,

where $U_{\mathrm{s}} \ell$ denotes the half-level transmit amplitude assuming $M_{\ell}$-ary $\mathrm{QAM}$ and $\sqrt{\xi_{\ell, k}}$ represents the positive square roots of the eigenvalues of the matrix $\mathbf{H}^{\mathrm{H}} \mathbf{H}$. Considering QAM constellations, the average transmit power $P_{\mathrm{s} \ell}$ per MIMO layer $\ell$ may be expressed as $[15,14]$

$P_{\mathrm{s} \ell}=\frac{2}{3} U_{\mathrm{s} \ell}^{2}\left(M_{\ell}-1\right)$.

Using parallel transmissions over $L \leq \min \left(n_{\mathrm{T}}, n_{\mathrm{R}}\right)$ MIMO layers, the overall mean transmit power becomes $P_{\mathrm{s}}=\sum_{\ell=1}^{L} P_{\mathrm{s} \ell}$, where the number of readily separable layers ${ }^{1}$ is limited by $\min \left(n_{\mathrm{T}}, n_{\mathrm{R}}\right)$. In order to transmit at a fixed data rate while maintaining the best possible integrity, i.e. BER, an appropriate number of MIMO layers has to be used, which depends on the specific QAM constellation size, as detailed in Table 1. The BER per MIMO layer $\ell$ and transmitted symbol block $k$ after SVD is given by [13]:

$P_{\mathrm{e}}^{(\ell, k)}=\frac{2\left(1-\frac{1}{\sqrt{M_{\ell}}}\right)}{\log _{2}\left(M_{\ell}\right)} \operatorname{erfc}\left(\sqrt{\frac{\xi_{\ell, k}}{2}} \cdot \frac{U_{\mathrm{s} \ell}}{U_{\mathrm{R}}}\right)$.

The resultant average BER per transmitted symbol block $k$ assuming different QAM constellation sizes

\footnotetext{
${ }^{1}$ It is worth noting that with the aid of powerful non-linear nearMaximum Likelihood (ML) sphere decoders it is possible to separate $n_{\mathrm{R}}>n_{\mathrm{T}}$ number of layers [16].
} 
Table 1

QAM constellations $M_{\ell}$ and corresponding $\alpha^{(\ell)}$

\begin{tabular}{lllll}
\hline & $M_{1}, \alpha^{(1)}$ & $M_{2}, \alpha^{(2)}$ & $M_{3}, \alpha^{(3)}$ & $M_{4}, \alpha^{(4)}$ \\
\hline $4 \mathrm{bit} / \mathrm{s} / \mathrm{Hz}$ & 16,1 & 0,0 & 0,0 & 0,0 \\
$\mathbf{4} \mathbf{b i t} / \mathbf{s} / \mathbf{H z}$ & $\mathbf{4 , 1 / 2}$ & $\mathbf{4 , 1 / 2}$ & $\mathbf{0 , 0}$ & $\mathbf{0 , 0}$ \\
$6 \mathrm{bit} / \mathrm{s} / \mathrm{Hz}$ & 64,1 & 0 & 0 & 0 \\
$\mathbf{6} \mathrm{bit} / \mathrm{s} / \mathrm{Hz}$ & $\mathbf{1 6}, \mathbf{2} / \mathbf{3}$ & $\mathbf{4 , 1 / 3}$ & 0 & 0 \\
$6 \mathrm{bit} / \mathrm{s} / \mathrm{Hz}$ & $4,1 / 3$ & $4,1 / 3$ & $4,1 / 3$ & 0 \\
$8 \mathrm{bit} / \mathrm{s} / \mathrm{Hz}$ & 256,1 & 0 & 0 & 0 \\
$8 \mathrm{bit} / \mathrm{s} / \mathrm{Hz}$ & $64,3 / 4$ & $4,1 / 4$ & 0 & 0 \\
$\mathbf{8} \mathbf{b i t} / \mathbf{s} / \mathbf{H z}$ & $\mathbf{1 6 , 1 / 2}$ & $\mathbf{4 , 1 / 4}$ & $\mathbf{4 , 1 / 4}$ & $\mathbf{0}$ \\
$8 \mathrm{bit} / \mathrm{s} / \mathrm{Hz}$ & $4,1 / 4$ & $4,1 / 4$ & $4,1 / 4$ & $4,1 / 4$ \\
\hline
\end{tabular}

becomes

$$
P_{\mathrm{e}}^{(k)}=\frac{1}{\sum_{\nu=1}^{L} \log _{2}\left(M_{\nu}\right)} \sum_{\ell=1}^{L} \log _{2}\left(M_{\ell}\right) P_{\mathrm{e}}^{(\ell, k)} .
$$

When considering time-variant channel SNR conditions, rather than an AWGN channel, having a fixed SNR, the BER can be derived by considering the different transmission block SNRs.

\section{Coded MIMO system}

BICM is constituted by the concatenation of an encoder, an interleaver and a mapper, which is extended here to a BICM-MIMO scheme, where different signal constellations are mapped appropriately to different SDM layers. Here, a sequence of information bits $\mathbf{i}$, organized into blocks of $N_{\mathrm{i}}$ bits, is encoded by a nonrecursive, non-systematic convolutional (NSC) code, resulting in the block $\mathbf{b}$ of $N_{\mathrm{b}}$ bits. The encoded bits are bit-interleaved by a random interleaver and stored in the vector $\tilde{\mathbf{b}}$. Afterwards, the NSC-encoded and interleaved bits are divided into sub-blocks $\left(\tilde{\mathbf{b}}_{1, k}, \tilde{\mathbf{b}}_{2, k}, \ldots, \tilde{\mathbf{b}}_{L, k}\right)$ consisting of

$R=\sum_{\ell=1}^{L} \log _{2} M_{\ell}$

bits. These sub-blocks are mapped onto the $L$ activated MIMO layers. Each symbol of a sub-block $\tilde{\mathbf{b}}_{\ell, k}$, consisting of $\log _{2} M_{\ell}$ bits, is mapped to the $M_{\ell \text {-ary }}$ QAM symbol $c_{\ell, k}$ according to the specific mapper used. The specific fraction of the data sequence $\mathbf{b}$ that is transmitted over the $\ell$ th SDM layer is given by the MIMO-layer-specific parameter $\alpha^{(\ell)}$ that can be calculated as follows

$\alpha^{(\ell)}=\frac{\log _{2} M_{\ell}}{R}$.

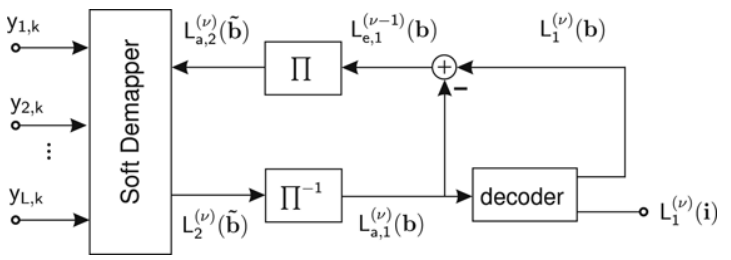

Fig. 1. Iterative demodulator structure.

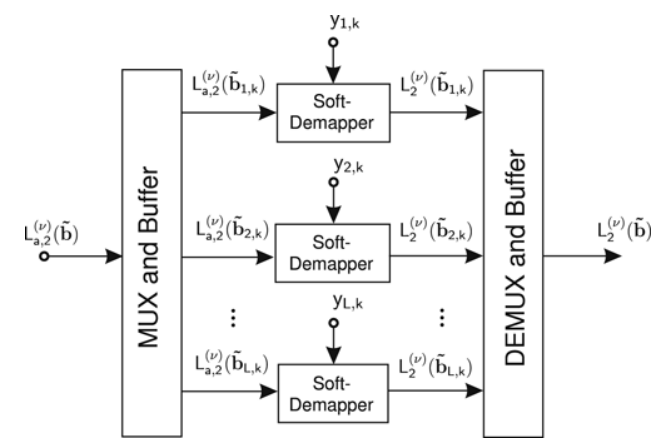

Fig. 2. Detailed soft-demapper demodulator structure.

The ratios $\alpha^{(\ell)} \in[0,1]$ must satisfy the condition

$$
\sum_{\ell=1}^{L} \alpha^{(\ell)}=1 .
$$

The resultant iterative demodulator structure is shown in Fig. 1. When using the iteration index $v$, the first iteration of $v=1$ commences with the soft-demapper delivering the $N_{\mathrm{b}} \log$-likelihood ratios (LLRs) $L_{2}^{(v=1)}(\tilde{\mathbf{b}})$ of the encoded and interleaved information bits, whose deinterleaved version $L_{\mathrm{a}, 1}^{(v=1)}$ (b) represents the input of the convolutional decoder depicted in Fig. 1. This channel decoder provides the estimates $L_{1}^{(\nu=1)}$ (i) of the original uncoded information bits as well as the LLRs of the $N_{\mathrm{b}}$ NSC-encoded bits in the form of

$L_{1}^{(\nu=1)}(\mathbf{b})=L_{\mathrm{a}, 1}^{(\nu=1)}(\mathbf{b})+L_{\mathrm{e}, 1}^{(\nu=1)}(\mathbf{b})$.

As seen in Fig. 1 and Eq. (12), the LLRs of the NSC-encoded bits consist of the receiver's input signal itself plus the extrinsic information $L_{\mathrm{e}, 1}^{(\nu=1)}(\mathbf{b})$, which is generated by subtracting $L_{\mathrm{a}, 1}^{(\nu=1)}(\mathbf{b})$ from $L_{1}^{(\nu=1)}(\mathbf{b})$. The appropriately ordered, i.e. interleaved extrinsic LLRs are fed back as a priori information $L_{\mathrm{a}, 2}^{(\nu=2)}(\tilde{\mathbf{b}})$ to the soft-demapper of Fig. 1 for the second iteration. Following the detailed structure of the soft-demapper in Fig. 2, the $N_{\mathrm{b}}$ LLRs $L_{2}^{(v)}(\tilde{\mathbf{b}})$ are composed of the sub-blocks $\left(L_{2}^{(v)}\left(\tilde{\mathbf{b}}_{1, k}\right), L_{2}^{(v)}\left(\tilde{\mathbf{b}}_{2, k}\right), \ldots, L_{2}^{(v)}\left(\tilde{\mathbf{b}}_{L, k}\right)\right)$, consisting of $R$ number of LLR elements. Each vector $L_{2}^{(v)}\left(\tilde{\mathbf{b}}_{\ell, k}\right)$ is generated by the soft-demapper from the MIMO channels' output $y_{\ell, k}$ and the a priori 


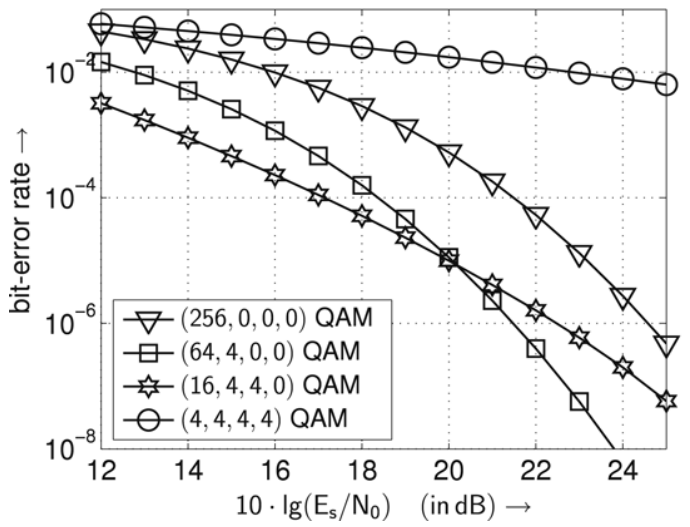

Fig. 3. Uncoded BERs when using the MIMO configurations introduced in Table 1 and transmitting 8 bit/s/Hz over non-frequency selective uncorrelated Rayleigh channels.

information $L_{\mathrm{a}, 2}^{(v)}\left(\tilde{\mathbf{b}}_{\ell, k}\right)$ provided by the channel decoder. After the first iteration, this a priori information emerges from the $N_{\mathrm{b}}$ LLRs $L_{\mathrm{a}, 2}^{(v)}(\tilde{\mathbf{b}})$.

\section{EXIT chart analysis}

The transmitted data sequence $\boldsymbol{B}$ is multiplexed onto the $L$ different MIMO layers, which results in the layerspecific sequence $\boldsymbol{B}_{\ell}, \ell=1,2, \ldots, L$. The stationary binary input sequence $\boldsymbol{B}_{\ell}=\left[B_{\ell, 1}, B_{\ell, 2}, \ldots, B_{\ell, k}, \ldots\right]$ consists of the r.v.s $B_{\ell, k}$, where the corresponding realizations $b_{\ell, k}$ have an index length of 1 bit and are taken from a finite alphabet $\mathcal{B}=\{0,1\}$. The sequence $\boldsymbol{C}_{\ell}=\left[C_{\ell, 1}, C_{\ell, 2}, \ldots, C_{\ell, k}, \ldots\right]$ mapped onto the $\ell$ th layer consists of the r.v.s $C_{\ell, k}$, where the corresponding realizations $c_{\ell, k}$ convey $\log _{2}\left(M_{\ell}\right)$ number of bits and are taken from a finite $M_{\ell}$-ary QAM alphabet $\mathcal{C}=\left\{0,1, \ldots, M_{\ell}-1\right\}$. The symbols $c_{\ell, k}$ are transmitted over independent channels, resulting in the received values $y_{\ell, k}$. The sequence $\boldsymbol{A}_{\ell}=$ $\left[A_{\ell, 1}, A_{\ell, 2}, \ldots, A_{\ell, k}, \ldots\right]$, having the corresponding realizations $a_{\ell, k}$ contains the $a$ priori LLR information passed to the demapper. EXIT charts visualize the input/output mutual information transfer between the data sequence $\boldsymbol{B}_{\ell}$ and the sequence $\boldsymbol{A}_{\ell}$ of $a$ priori LLR information at the input of the soft-demapper, as well as between $\boldsymbol{B}_{\ell}$ and the sequence $\boldsymbol{E}_{\ell}$ of the extrinsic LLR, respectively. Denoting the mutual information between two r.v.s $X$ and $Y$ as $I(X ; Y)$ we may define the quantities $I_{\ell, A}=I\left(\boldsymbol{A}_{\ell} ; \boldsymbol{B}_{\ell}\right)$ as well as $I_{\ell, E}=I\left(\boldsymbol{E}_{\ell} ; \boldsymbol{B}_{\ell}\right)$ for a given sequence $\boldsymbol{B}_{\ell}$, where $I_{\ell, A}$ represents the average a priori information and $I_{\ell, E}$ the average extrinsic information, respectively [17]. The EXIT characteristic $T$ of the soft-demapper in Fig. 1 is given by $I_{\ell, E}=T\left(I_{\ell, A}, \rho\right)$, where $\rho$ represents the SNR of the communication channel. Analyzing the outer decoder in a serially concatenated scheme $T$ does not depend on $\rho$. The EXIT chart is now obtained by plotting the transfer characteristics $T$ for both the demapper and the decoder of Fig. 1 within a single diagram [12]. The number of transmitted bits per symbol and block, including all $L$ SDM MIMO layers, results in $R$ bits, as defined in (9). Together with the MIMO-layer-specific parameter $\alpha^{(\ell)}$ defined in (10), the fraction of the data sequence $\boldsymbol{B}$ that is transmitted over the $\ell$ th layer, i. e. $\boldsymbol{B}_{\ell}$ can be defined. Hence, the mutual information for a given sequence $\boldsymbol{B}$ and the extrinsic LLR $\boldsymbol{E}$ at the output is the $\alpha^{(\ell)}$. weighted linear combination of the layer-specific EXIT function $I\left(\boldsymbol{E}_{\ell} ; \boldsymbol{B}_{\ell}\right)$ and is obtained by

$I(\boldsymbol{E} ; \boldsymbol{B})=\sum_{\ell=1}^{L} \alpha^{(\ell)} I\left(\boldsymbol{E}_{\ell} ; \boldsymbol{B}_{\ell}\right)$.

Beneficial values of $\alpha^{(\ell)}$ may be chosen by ensuring that there is an open EXIT tunnel between the soft-demapper transfer characteristic and the decoder transfer characteristic at a given $E_{\mathrm{s}} / N_{0}$ value that is close to the channel's capacity bound.

\section{Results}

\subsection{Uncoded system}

Assuming predefined QAM constellation sizes, a fixed total throughput can be guaranteed for each data block. The corresponding calculated BER curves are depicted in Fig. 3 for the different QAM constellation sizes and MIMO configurations of Table 1 , when transmitting at a bandwidth efficiency of $8 \mathrm{bit} / \mathrm{s} / \mathrm{Hz}$, assuming a Nyquist roll-off factor of 0.5. Assuming an equal transmit power for the activated MIMO layers, it turns out that not all MIMO layers have to be activated in order to achieve the best BERs. More explicitly, our goal is to find that specific combination of the QAM mode and the number of MIMO layers, which gives the best possible BER performance at a given fixed bit/s/Hz bandwidth efficiency. The $E_{\mathrm{S}} / N_{0}$ value required by each scheme at BER $10^{-4}$ was extracted from computer simulations and the best systems are shown in bold in Table 1. Further improvements in terms of the BER are possible by using unequal power allocation (PA). However, as shown in [18], unequal $\mathrm{PA}$ is only effective in conjunction with finding the optimum number of activated MIMO layers.

\subsection{Coded system}

Using the half-rate, constraint-length $K=3$ NSC code with the generator polynomials of $(7,5)$ in octal 


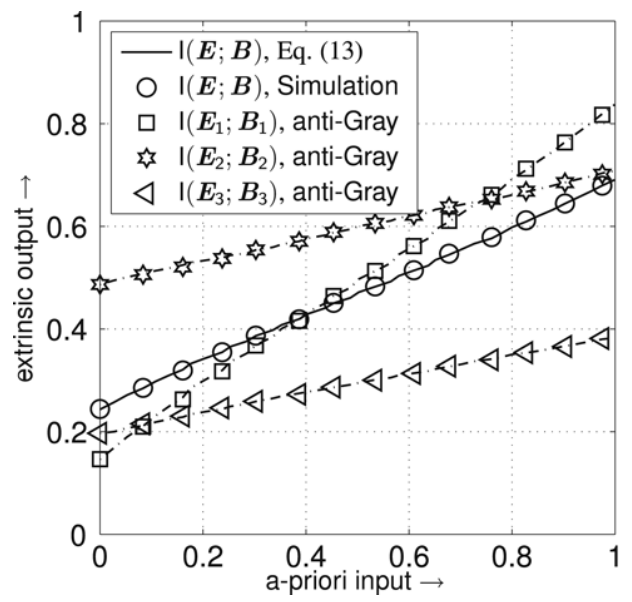

Fig. 4. Soft-Demapper transfer characteristic using anti-Gray mapping on all used MIMO layers and the $(16,4,4,0)$ QAM constellation at $10 \log _{10}\left(E_{\mathrm{S}} / N_{0}\right)=0 \mathrm{~dB}$.

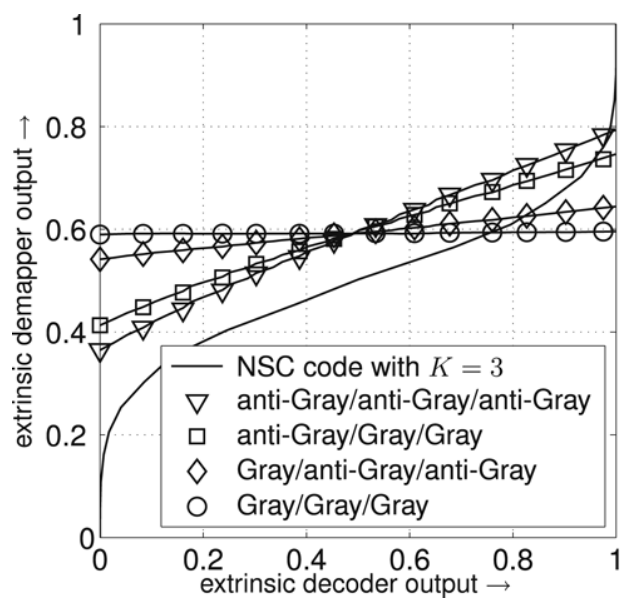

Fig. 5. EXIT chart for an effective throughput of $4 \mathrm{bit} / \mathrm{s} / \mathrm{Hz}$ and the $(16,4,4,0)$ QAM constellation at $10 \log _{10}\left(E_{\mathrm{S}} / N_{0}\right)=2 \mathrm{~dB}$.

notation, the BER performance is analyzed for an effective throughput of $4 \mathrm{bit} / \mathrm{s} / \mathrm{Hz}$. In addition to the number of bits per symbol and the number of activated MIMO layers, the achievable performance of the iterative decoder is substantially affected by the specific mapping of the bits to both the QAM symbols as well as to the MIMO layers. While the employment of the classic Gray-mapping is appropriate in the absence of a priori information, the availability of a priori information in iterative receivers requires an exhaustive search for finding the best non-Gray - synonymously also referred to as anti-Gray - mapping scheme [2].

Assuming predefined QAM constellation sizes and the corresponding $\alpha^{(\ell)}$, the soft-demapper's EXIT characteristic is given by combining the single MIMO

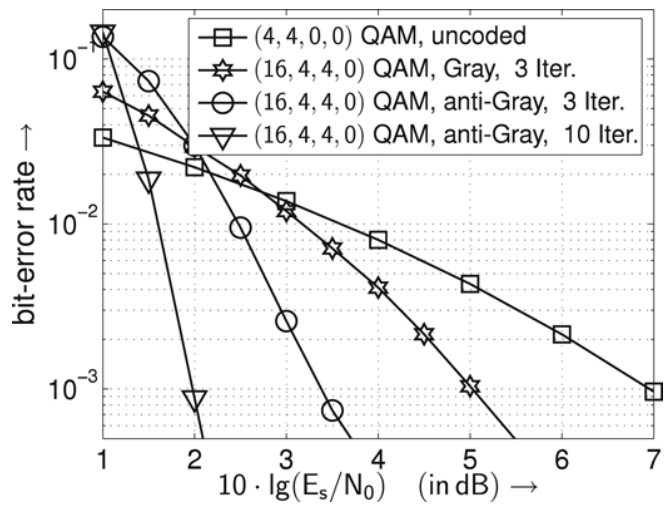

Fig. 6. BER assuming Gray or anti-Gray mapping schemes on all activated MIMO layers for an effective user throughput of $4 \mathrm{bit} / \mathrm{s} / \mathrm{Hz}$.

layers' EXIT characteristics using the parameter $\alpha^{(\ell)}$, as depicted in Fig. 4. A mapping scheme optimized for perfect a priori information has usually a poor performance, when there is no a priori information. However, when applying iterative demapping and decoding, large gains can be achieved as long as the reliability of the a priori information increases upon increasing the number of iterations. As depicted in Fig. 5, the maximum iteration gain can only be guaranteed, if anti-Gray mapping is used on all activated MIMO layers. However, observed by comparing the EXIT chart results of Fig. 5, the overall performance is strongly influenced by the most susceptible MIMO layer, which is here the MIMO layer transmitting $4 \mathrm{bit} / \mathrm{s} / \mathrm{Hz}$. The BER performance is characterized in Fig. 6 for the half-rate, constraint-length $K=3$ NSC code assuming an effective user throughput of $4 \mathrm{bit} / \mathrm{s} / \mathrm{Hz}$. The BER investigations using the NCS code are based on the best uncoded schemes of Table 1 . The information word length is 1000 bits and a random interleaver is applied. The influence of the Gray versus anti-Gray mapping is clearly visible in Fig. 6.

\section{Conclusion}

In analogy to BICIM, we introduced a MIMOBICM scheme, where different signal constellations and mappings were used within a single codeword. The proposed system includes an adaptation of the transmit parameters. EXIT charts are used for analyzing and optimizing the convergence behaviour of iterative demapping and decoding. The choice of the number of bits per symbol and the number of MIMO layers combined with error correcting codes substantially affects the performance of a MIMO system, suggesting that not all MIMO layers have to be activated in order to achieve the best BERs. 


\section{References}

[1] G. Caire, G. Taricco, E. Biglieri, Bit-interleaved coded modulation, IEEE Transactions on Information Theory 44 (3) (1998) 927-946.

[2] J.A. Chindapol, A. Ritcey, Design, analysis, and performance evaluation for BICM-ID withsquare QAM constellations in rayleigh fading channels, IEEE Journal on Selected Areas in Communications 19 (5) (2001) 944-957.

[3] F. Schreckenbach, G. Bauch, Bit-interleaved coded irregular modulation, European Transactions on Telecommunications 17 (2) (2006) 269-282.

[4] M.R. McKay, I.B. Collings, Capacity and performance of MIMO-BICM with zero-forcing receivers, IEEE Transactions on Communications 53 (1) (2005) 74-83.

[5] S.H. Mueller-Weinfurtner, Coding approaches for multiple antenna transmission in fast fading and OFDM, IEEE Transactions on Signal Processing 50 (10) (2002) 2442-2450.

[6] B.S. Krongold, K. Ramchandran, D.L. Jones, Computationally efficient optimal power allocation algorithms for multicarrier communications systems, IEEE Transactions on Communications 48 (1) (2000) 23-27.

[7] J. Jang, K.B. Lee, Transmit power adaptation for multiuser ofdm systems, IEEE Journal on Selected Areas in Communications 21 (2) (2003) 171-178.

[8] R.F.H. Fischer, J.B. Huber, A New Loading Algorithm for Discrete Multitone Modulation, in: IEEE Global Telecommunications Conference, GLOBECOM, London, 1996, pp. 724-728.

[9] C.S. Park, K.B. Lee, Transmit power allocation for BER performance improvement in multicarrier systems, IEEE Transactions on Communications 52 (10) (2004) 1658-1663.

[10] Z. Zhou, B. Vucetic, M. Dohler, Y. Li, MIMO systems with adaptive modulation, IEEE Transactions on Vehicular Technology 54 (5) (2005) 1073-1096.

[11] I. Kalet, Optimization of linearly equalized QAM, IEEE Transactions on Communications 35 (11) (1987) 1234-1236.

[12] S. ten Brink, Convergence behavior of iteratively decoded parallel concatenated codes, IEEE Transactions on Communications 49 (10) (2001) 1727-1737.

[13] A. Ahrens, C. Lange, Transmit power allocation in SVD equalized multicarrier systems, International Journal of Electronics and Communications (AEÜ) 61 (1) (2007) 51-61.

[14] J.G. Proakis, Digital Communications, McGraw-Hill, Boston, 2000.

[15] G.D. Forney, R.G. Gallager, G.R. Lang, F.M. Longstaff, S.U. Qureshi, Efficient modulation for band-limited channels, IEEE Journal on Selected Areas in Communications 2 (5) (1984) 632-647.

[16] L. Hanzo, T. Keller, OFDM and MC-CDMA, Wiley, New York, 2006.

[17] A. Ahrens, V. Kuehn, Analysis of SVD-aided, iteratively detected spatial division multiplexing using exit charts, in: 12th International OFDM-Workshop, Hamburg, 29-30 August 2007, pp. 271-275.

[18] A. Ahrens, V. Kühn, T. Weber, Iterative detection for spatial multiplexing with adaptive power allocation, in: 7th International Conference on Source and Channel Coding, SCC, Ulm, 14-16 January 2008.

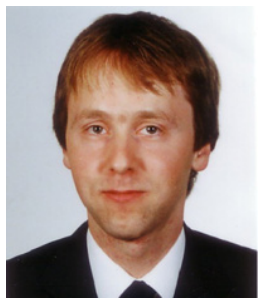

Andreas Ahrens was born in Wismar, Germany, in January 1971. He received the Dipl.Ing. degree in electrical engineering from the University of Rostock in 1996. Since then he has been with the Institute of Communications Engineering of the University of Rostock, from which he received the Dr.-Ing. and Dr.-Ing. habil. degrees in 2000 and 2003, respectively. His research focusses on equalization and multicarrier transmission for both wireline and wireless communication as well as on multiple antenna systems and iterative detection. He is a member of the IEEE.

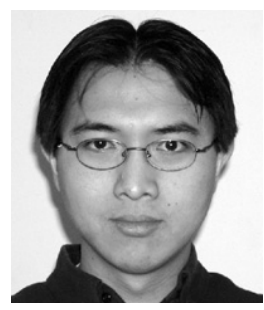

Soon Xin Ng (S'99-M'03) received the B.Eng. degree (First class) in electronics engineering and the Ph.D. degree in mobile communications from the University of Southampton, Southampton, UK, in 1999 and 2002, respectively. From 2003 to 2006, he was a postdoctoral research fellow at the University of Southampton working on collaborative European research projects known as SCOUT, NEWCOM and PHOENIX. Since August 2006, he has been a lecturer in wireless communications at the University of Southampton. His research interests are mainly in adaptive coded modulation, channel coding, turbo coding, space-time coding, and joint source and channel coding. He has published numerous papers and coauthored a book in this field.

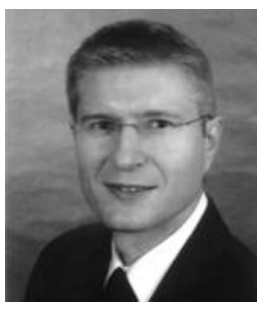

Volker Kühn received the diploma degree in electrical and electronics engineering from the University of Paderborn in 1993. From 1994 to 1998 he was with the Department of Communications Engineering at the University of Paderborn. After receiving the Ph.D. degree in 1998, he joined the Department of Communications Engineering at the University of Bremen where he wrote his postdoctoral thesis. From 2005 to 2006 , he was with Qualcomm CDMA Technologies GmbH in Nuremberg, Germany. In 2006, he became a Professor for Communications Engineering at the University of Rostock, Germany. Dr. Küehn is author/coauthor of three text books and numerous conference and journal contributions. His main field of interest is mobile radio communications, especially the topics error correcting codes, multiple antenna systems, iterative detection and distributed coding strategies.

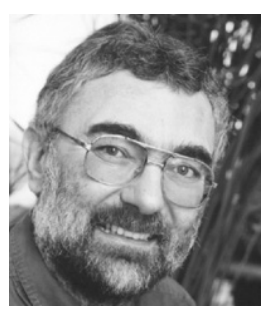

Lajos Hanzo, Fellow of the Royal Academy of Engineering, received his first-class degree in electronics in 1976 and his doctorate in 1983. In 2004 he was awarded the Doctor of Sciences (D.Sc.) degree by the University of Southampton, UK. During his career in telecommunications he has held various research and academic posts in Hungary, Germany and the UK. Since 1986 he has been with the Department of Electronics and Computer Science, University of Southampton, UK, where he holds the chair in telecommunications. He has coauthored 12 books, totalling 9000 pages on mobile radio communications, published more than 600 research papers, has acted as TPC Chair of numerous major IEE and IEEE conferences, presented various keynote lectures and has been awarded a number of distinctions. Currently he heads an academic research team, working on a range of research projects in the field of wireless multimedia communications sponsored by industry, the Engineering and Physical Sciences Research Council (EPSRC) 
UK, the European IST Programme and the Mobile Virtual Centre of Excellence (VCE), UK. He is an enthusiastic supporter of industrial and academic liaison and he offers a range of industrial courses. Lajos is also an IEEE Distinguished Lecturer of both the Communications as well as the Vehicular Technology Society, a Fellow of both the IEEE and the IEE. He is an editorial board member of the Proceedings of the IEEE and a Governor of the IEEE VT Society. Currently he acts as the Editor-in-Chief of the IEEE Press. For further information on the research in progress and associated publications, please refer to http://www-mobile.ecs.soton.ac.uk 